\title{
An Improved Quantum Evolution Algorithm and Performance Analysis
}

\author{
Zhang Yi, Yang Xiuxia \\ Department of Control Engineering \\ Naval Aeronautical and Astronautical University \\ Yantai, China \\ changyee@tom.com
}

\author{
Zhou Shaolei \\ Department of Control Engineering \\ Naval Aeronautical and Astronautical University \\ Yantai, China
}

\begin{abstract}
To tackle the shortcoming of deficient using of feedback information in quantum-inspired evolutionary computing and catastrophe such as quantum mutation, the improved quantum-inspired evolutionary algorithm (IQEA) is proposed. The crossover and mutation operators of genetic algorithm are introduced to the quantum evolution algorithm, at the same time, the better individuals information is applied to the generation of the population applying the probability evaluation model of the estimation of distribution algorithm. Theory analyze and test function simulation experiments show that the improved quantum genetic algorithm is characterized by rapid convergence, excellent robustness and so on.
\end{abstract}

Keywords-Multiple quantum computation; quantum-inspired evolutionary algorithm; genetic optimization; estimation of distribution

\section{INTRODUCTION}

Quantum computation is a new computation model which uses the quantum superimpose and coherence to complete the parallel computation, which has many advantages on the traditional optimization approaches such as hill-climbing and conjugate gradient methods, the quantum evolutionary algorithm (QEA ) does not depend on the problem's accurate modal and therefore an QEA is widely adaptable. In the past decade years, QEA has a great development and different kinds of QEAs have been designed[1-4]. Narayanan first offers a genetic quantum algorithm (GQA)[1]. Combining the quantum computation and the traditional intelligent algorithm, the quantum particle swarm optimization algorithm is provided.

In the past decade years, different kinds of QEAs have been designed [1-3]. Since the QEA was presented, it has attracted a wide attention in different fields and has been applied in nonlinear, discontinuous and multimodal optimization problems such as machine learning and artificial neural network training.

Quantum genetic algorithm(QGA) is characterized by rapid convergence and global search capability, simultaneously. QGA is based on the concept and principles of quantum computing such as qubits and a linear superposition of states. One individual of QGA can represent many states at the same time, and there are weak relationships between individuals since each individual is determined by current best solution and its probability, that is, the history of individual, up to date. Because of this reason, QGA is suitable for parallel structure [4].
To tackle the shortcoming of deficient using of feedback information in quantum-inspired evolutionary computing, the crossover and mutation operators is introduced to the quantum evolution algorithm, at the same time, the better individuals information is applied to the generation of the population applying the probability evaluation model of the estimation of distribution algorithm. The hybrid quantuminspired evolutionary algorithm (HQEA ) is proposed in this paper and the algorithm performance is analyzed. Test function is used to test the algorithm.

\section{ALGORITHM DESIGN}

Quantum-inspired genetic algorithm (QGA) is based on the concepts of qubits and superposition of states of quantum mechanics. The smallest unit of information stored in a twostate quantum computer is called a quantum bit or qubit. A qubit may be in the ' 1 ' state, in the ' 0 'state, or in any superposition of the two. The state of a qubit can be represented as

$$
|\psi\rangle=\alpha|0\rangle+\beta|1\rangle
$$

where $\alpha$ and $\beta$ are complex numbers that specify the probability amplitudes of the corresponding states. $|\alpha|^{2}$ gives the probability that the qubit will be found in ' 0 ' state and $|\beta|^{2}$ gives the probability that the qubit will be found in the ' 1 ' state. Normalization of the state to unity guarantees

$$
|\alpha|^{2}+|\beta|^{2}=1
$$

If there is a system of $m$-qubits, the system can contain information of $2^{m}$ states. However, in the act of observing a quantum state, it collapses to a single state.

It is possible to use a number of different representations to encode the solutions onto chromosomes in evolutionary algorithm. The classical representations can be broadly classified as: binary, numeric, and symbolic. QGA uses a novel representation that is based on the concept of qubits. One qubit is defined with a pair of complex numbers $(\alpha, \beta)$ as

$\left[\begin{array}{l}\alpha \\ \beta\end{array}\right]$

which is characterized by (1) and (2). And a m-qubits representation is defined as 


\section{$\left[\begin{array}{c|c|c|c}\alpha_{1} & \alpha_{2} & \cdots & \alpha_{m} \\ \beta_{1} & \beta_{2} & \cdots & \beta_{m}\end{array}\right]$}

Where $\left|\alpha_{i}\right|^{2}+\left|\beta_{i}\right|^{2}=1 \quad, \quad i=1,2, \cdots m \quad$. This representation has the advantage that it is able to represent a superposition of states.

QGA is a probabilistic algorithm which is similar to genetic algorithm. QGA maintains a population of qubit chromosomes. Each individual of the population has its own adaptive value, and then do the genetic manipulation of simulated natural evolution, such as choose, crossover and mutation, repeating the manipulation until the problem is solved. Crossover and mutation can increase the pattern.

QGA maintains a population of qubit chromosomes, $Q(t)=\left\{q_{1}^{t}, q_{2}^{t}, \cdots, q_{n}^{t}\right\}$ at generation $t$, where $n$ is the size of population, and $q_{j}^{t}$ is a qubit chromosome defined as

$$
q_{j}^{t}=\left[\begin{array}{c|c|c|c}
\alpha_{1}^{t} & \alpha_{2}^{t} & \ldots & \alpha_{m}^{t} \\
\beta_{1}^{t} & \beta_{2}^{t} & \cdots & \beta_{m}^{t}
\end{array}\right]
$$

Where $m$ is the number of qubits, i.e., the string length of the qubit chromosome, and $j=1,2, \cdots, n$.

The detail operation can reference to [2].

Traditional evolutionary algorithm achieves the population evolution based on the genetic manipulation for the every individual in the population (crossover and mutation, etc.) and establishes the mathematical model from the "micro" level. But estimation of distribution algorithm (EDA) directly describes the evolution trend of the population through the establishment of a mathematical model based on the whole population from the "macro" level in biological evolution.

In EDA, there is no traditional genetic operation such as crossover and mutation, but the learning and sampling of the probabilistic model. EDA describe the distribution of the candidate solutions in space through a probability model, uses statistical learning tools from the perspective of the macro group to establish a probabilistic model to describe the distribution of solutions, and then take a random sampling of the probabilistic model to generate new populations, repeat it and achieve the optimization of the population[5-8]. EDA can utilize the better individuals of the population, while not only the best one.

The establishment of the probability evaluation model is the core of EDA, which is the key of the model parameter optimization. The probability model is composed of the structure and a set of local generalized probability density. Suppose $\boldsymbol{X}=\left(\boldsymbol{X}_{1}, \boldsymbol{X}_{2}, \cdots, \boldsymbol{X}_{\boldsymbol{i}}, \cdots, \boldsymbol{X}_{n}\right)$ is a set of random variant, generate the evaluation individual $x=\left(x_{1}, x_{2}, \cdots, x_{i}, \cdots, x_{n}\right)$ randomly. Let $\boldsymbol{S}$ denotes the structure of $\boldsymbol{X}, \boldsymbol{\rho}(\boldsymbol{x})$ denotes the local generalized probability density of $\boldsymbol{X}$. At present, the study of discrete EDA is relative mature, the probability model is expressed by Bayes network.

According to the complexity of probability model, EDA can be classified to the independent variant distribution evaluation, bivariate and multivariable correlation distribution evaluation. The more complex of the variant relationship is, the more difficulty of the calculation. If the relationship of every variant is decomposed and select the independent variant distribution UDMA (Univariate marginal distribution algorithm) to complete the parameter optimum, the probability distribution is

$$
\rho\left(x \mid \theta_{s}\right)=\prod_{i=1}^{n} \rho\left(x_{i}, \theta_{i}\right)
$$

Where $\boldsymbol{\theta}_{\boldsymbol{s}}=\left(\boldsymbol{\theta}_{1}, \boldsymbol{\theta}_{2}, \cdots, \boldsymbol{\theta}_{i}, \cdots, \boldsymbol{\theta}_{\boldsymbol{n}}\right)$ is the limited parameter sets.

Combing the merits of the QGA and EDA, the structure of improved quantum evolution algorithm(IQEA) is described on the following:

begin

$t \leftarrow 0$

initialize $Q(t)$

make $^{P(t)}$ by observing $Q(t)$ states

evaluate $P(t)$

store the best solution among $P(t)$

while (not termination-condition) do

begin

$t \leftarrow t+1$

half of the individuals make $P(t)$ by observing $Q(t-1)$ states

individuals crossover

evaluate $P(t)$

half of the individuals(the above individuals) update $Q(t)$ using quantum gates

half of the individual update $Q(t)$ using the probability evaluation model of the better individuals

store the best solution among $P(t)$

end

end

\section{EXPERIMENT}

Select De Jong function as the algorithm optimization examples:

De Jong function:

$$
\begin{aligned}
& \max f\left(x_{1}, x_{2}\right)=100\left(x_{1}^{2}-x_{2}\right)^{2}+\left(1-x_{1}\right)^{2} \\
& \text { sub } \quad-2.048 \leq x \leq 2.048
\end{aligned}
$$

Which is a two-dimension function and has a global maximum $f(-2.048,-2.048)=3905.926$. Although this function is a single peak function, it is ill-conditioning and is difficult to maximize. Through 50 times simulation to evaluate optimization ability of the quantum-inspired genetic algorithm (QGA), Genetic Algorithm (GA) and improved quantum evolution algorithm(IQEA).

Run each optimization algorithm 50 times independently. The best fitness curve of the three optimization methods are given in figure 1 . As is shown in the results, since the best individual is not be learned, GA evolves the slowest and not 
fit to find the global optimal solution. For the introduce of quantum evolution, the diversity of the population is increased, QGA evolves rapidly, but it don't fully use the evolution information. Since IQEA applies the probability evaluation model of the estimation of distribution algorithm, evolves from the macro and micro view of population, it evolves the fastest and the easiest to find global extreme point.

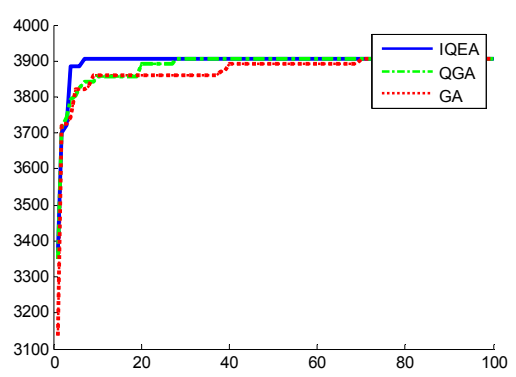

Figure 1. Evolution curve of the three optimization methods

\section{CONCLUSION}

The crossover and mutation operators is introduced to the quantum evolution algorithm, at the same time, the better individuals information is applied to the generation of the population applying the probability evaluation model of the estimation of distribution algorithm. The improved algorithm is convergent quickly and the diversity of the population is retained. Simulation results show the effectiveness of the algorithm.

\section{REFERENCES}

[1] Narayanan A, M ooreM. Quantum -inspired genetic algorithm [A ].Proceedings of IEEE International Conference on Evolutionary Computation[ C ], Piscataway: IEEE Press, 1996, 61-66.

[2] K.-H. Han and J.-H. Kim, Genetic Quantum Algorithm and its Application to Combinatorial Optimization Problem, in Proceedings of the 2000 Congress on Evolutionary Computation, pp. 1354-1360, July, 2000.

[3] Han K H, Kim J H. Genetic quantum algorithm and its application to combinatorial optimization problems. Proc of IEEE Conference on Evolutionary Computation [ C ] , Piscataway: IEEE Press, 2000, 1354-1360.

[4] Han K H, Park K H. Parallel quantum-insp ired genetic algorithm for combinatorial optimization problems. Proc of the IEEE Conference on Evolutionary Computation [ C ] , Piscataway: IEEE Press, 2001, 1442-1429.

[5] Larrinaga P, Lozano J A. Estimation of Distribution Algorithms. A New Tool for Evolutionary Computation. Boston: Kluwer Academic Publishers, 2002

[6] Pelikan M, Goldberg D E, Lobo F. A Survey of Optimization by Building and Using Probabilistic Models. IlliGAL Report No. 99018, University of Illinois at Urbana-Champaign, Illinois Genetic Algorithms Laboratory, Urbana, Illinois, 1999

[7] Pelikan M, Goldberg D E, Caniu-Pes E. Linkage Problem Distribution Estimation and Bayesian Networks. IlliGAL Report No. 98013, Univeraity of Illinois at Urbana--Champaign, Illinois Genetic Algorithms Laboratory, Urbana, Illinois, 1998

[8] Ye Jun, Lv Qiang. Application of Estimation of Distribution Algorithm Based On Transition Probability to P-Median Problem. Computer Applications and Software, 2008(25), 11(in Chinese) 